\title{
CARCASS QUALITY OF CRIOLLO CORDOBES AND ANGLO NUBIAN SUCKLING KIDS. EFFECTS OF AGE AT SLAUGHTER
}

\author{
CARACTERÍSTICAS DE LA CANAL DE CABRITOS DE LAS RAZAS CRIOLLO \\ CORDOBÉS Y ANGLO NUBIANA. EFECTO DE LA EDAD AL SACRIFICIO
}

Peña, F. ${ }^{1 *}$, Bonvillani, A. ${ }^{2}$, Morandini, M. ${ }^{2}$, Freire, ${ }^{2} .{ }^{2}$, Domenech, $V .{ }^{1}$ and García, A. ${ }^{1}$

1'Departamento de Producción Animal. Universidad de Córdoba.14014 Córdoba. España. *pa1peblf@uco.es ${ }^{2}$ Departamento de Producción Animal. Fac. Agronomía y Veteriaria. Universidad de Rio Cuarto. Argentina.

\section{AdDitionAl KeYWORDS}

Goat.

\section{SUMMARY}

The objective of this study was to assess the carcass characteristics of two goat breed types reared under extensive system. Forty male kids, 20 Criollo Cordobes and 20 Anglo Nubian, were used. The animals were slaughtered at $60 \pm 2$ days old (group I) and at $90 \pm 3$ days old (group II). The carcass yield and fatness was significantly higher in Anglo Nubian than in Criollo Cordobes kids. Breed and age at slaughter significantly affect carcass conformation, some carcass measures and indices, and non-carcass components.

\section{RESUMEN}

Se estudiaron las características de la canal de cabritos lactantes de las razas Criollo Cordobés y Anglo Nubiana, utilizando 20 machos de cada raza. Los animales se sacrificaron a dos edades: $60 \pm 2$ días (grupo I) y $90 \pm 3$ días (grupo II). Los rendimientos canal y el grado de engrasamiento fueron superiores en Anglo Nubiana. La raza y la edad al sacrificio tuvieron un efecto significativo en la valoración subjetiva, en las medidas e índices de la canal y en el quinto cuarto.

\section{INTRODUCTION}

Currently, the production of goat meat is located in the Argentine northwest, being Criollo Cordobes goat (70\%) the most common breed of goats in this region. They are bred mostly for meat production. The male kids of Anglo Nubian breed, the most

Recibido: 23-4-09. Aceptado: 22-7-09.

\section{Palabras clave adicionales \\ Cabras.}

common dairy breed, are destined for meat production (Maubecin, 1973).

Argentine goat meat production is based mainly on extensive systems by grazing on natural prairies with shrubs and forest, and scarce o non-existent feed supplementation (Maubecin, 1976). Currently, the demand for meat from goats has increased and meat from young animals is considered a delicacy. For this reason, kids are slaughtered at lowest age/weight (30-65 days old and 6-12 $\mathrm{kg}$ liveweight, Arias and Alonso, 2002). The information on the carcass characteristics in suckling kids from Criollo Cordobes and Anglo Nubian goat breeds is not well documented. Several studies have previously been carried out on carcass traits of Creole goats (Maubecin, 1976; Rossanigo et al., 1995), and very few on the Criollo Cordobes (De Gea et al., 2005b). Also, there is no available information about the carcass characteristics of Anglo Nubian kids slaughtered a low weight.

\section{MATERIAL AND METHODS}

The animals, suckling intact males of the Criollo Cordobes (CC) and Anglo Nubian (AN) breeds, were purchased from two local farmers. The production system was extensive: the adults were fed on pastures 
without concentrate supplements, and the kids were nursing milk from their mothers and left to graze with their dams. At birth, 20 males from each breed were randomly allocated to two groups: I (slaughtered at $60 \pm 2$ days old) and II (slaughtered at $90 \pm 3$ days old). When kids reached the slaughter age, they were transported to the abattoir ( 5 km away). Slaughter live weight (SLW) was recorded immediately prior to slaughter. Each group was slaughtered in 1 day, after a 12-h fast. Kids were stunned with a captive bolt pistol and slaughtered by bleeding. Hot carcass weight (HCW) and weight of non carcass components (blood, head, skin, feet, gastro-intestinal tract, some visceral organs, fat depots) were recorder within $1 \mathrm{~h}$ post-mortem. Tail, thymus, lateral portion of the diaphragm, kidneys, perinephric and pelvic fats, and testes were retained in carcass. After chilling $\left(24 \mathrm{~h}\right.$ at $\left.4^{\circ} \mathrm{C}\right)$, cold carcass weights (CCW) and empty body weights (EBW, live weight minus the weight of digesta) were recorded. Then, each carcass was evaluated for conformation (scale from poor $=1$ to $\operatorname{good}=3$ ), subcutaneous fatness (scale from $1=$ low to $5=$ very high $)$, meat colour $(1=$ pale; $2=$ pink; $3=$ red $)$ and fat colour $(1=$ white; $2=$ cream; $3=$ yellow) using the scoring system suggested by Colomer-Rocher et al. (1987). The internal fat cover was subjectively assessed using a scoring system, taking the pelvic-renal fat as a whole $(1=$ low; $2=$ medium; $3=$ high $)$. The carcass percentages were calculated as follows:

HCW/SLW $(\%)=($ hot carcass weight $\times 100) /$ slaughter live weight;

HCW/EBW $(\%)=($ hot carcass weight $\times 100) /$ empty body weight;

CCW/SLW $(\%)=($ cold carcass weight $\times 100) /$ slaughter live weight;

CCW/EBW $(\%)=($ cold carcass weight $\times 100) /$ empty body weight.

Objective carcass conformation and indices were measures as described in
Palsson (1939) and Boccard et al. (1958). The following measurements and indices were scored: internal carcass length (L), leg length (F), buttock width (G), buttock perimeter(BG), thoracic perimeter(PT), chest depth (Th), thorax width (Wr), maximum rib width (Wth), carcass compactness (HCW/ $\mathrm{L} ; \mathrm{CCW} / \mathrm{L})$, leg compactness (G/F), L/G, Wr/ Th, Wth/Th, Th/L, Th/G, and L/PT. The rib eye width (A) and depth (B) were measured with a calliper and ribeye area was obtained using the following equation:

rib eye area $=(\mathrm{A} / 2) \times(\mathrm{B} / 2) \times \pi($ Yañez et al. 2006).

After chilling, the left half of each carcass (CLSW) was weighed and physically dissected into five primal cuts (shoulder, leg, ribs, flank and neck) according to Colomer-Rocher et al. (1987). Primal cuts were weighed and grouped into three categories: extra (leg and ribs), first (shoulder) and second (neck and flank). The tail, testes, kidney, pelvic-perirenal fat in the carcass left side were removed before jointing and weighed. The shoulder was separated into dissectible fat (subcutaneous+intermuscular), muscle, bone and other tissue (major blood vessels, ligaments, tendons and fascias).

Statistical analysis of data were performed using the GLM procedure of SAS (SAS, 1997), in a 2 x 2 factorial arrangement with breed-type (CC and AN) and age (group I and group II) as main effects. Empty body weight was used as a covariate in the analysis of carcass measurements, non-carcass components and dressing percentages; hot carcass weight was used as a covariate in the analysis of primal cuts and dissectible tissues. Least squares means (LSM) and their standard errors were computed and tested for treatments differences. No significant interactions were noted for the parameters evaluated in the present study, therefore, only main effects are presented and discussed. The analysis of the 


\section{CARCASS QUALITY OF SUCKLING GOAT KIDS}

qualitative variables was conducted using bivaried contingency tables, and chi-square tests.

\section{RESULTSANDDISCUSSION}

Average weight values at slaughter from CC and AN kids (table I) are comparable to those reported for suckling kids in Argentina (De Gea et al., 2005b; Domingo et al., 2008; Zimmerman et al., 2008). The EBW, $\mathrm{HCW}$ and $\mathrm{CCW}$ increased with the increase of the age at slaughter.

Chilling losses (table I) were higher than those reported by Marichal et al. (2003) in kids slaughtered at similar live weight. With the age at slaughter increased, percentages of chilling losses were slightly greater. The increase of body surface/fatness ratio, due to low deposition of subcutaneous fat, might be the main reason for these results.

Values obtained from carcass percentage are comparable to those obtained in Creole kids and their crosses (Zimmerman et al.,
2008; Domingo et al., 2008), and within those found in a different genotypes at similar carcass kid weight (Santos et al., 2007). The lower dressing obtained by Meneses et al. (2004) can be explained by their greater body weight $(30 \mathrm{~kg})$ and age ( 6 months), and therefore the further development of the digestive tract ( $12.5 \%$ vs. $7-8 \%)$. The dressing percentage was higher $(\mathrm{p}<0.05)$ in AN kids than CC kids $(50-58 \%$ vs. 47-55\%). These differences can be attributed to a higher milk production from their mothers and therefore their greater growth rate. The lower visceral percentage (table V) and the higher degree of fat cover (table IV) from carcasses of AN kids could be the main reasons for these differences between breeds.

The carcass percentages did not change with the increase in body weight, which disagree with the findings of Owen et al. (1982). Marichal et al. (2003) recorded an increase in the dressing percentage in kids slaughtered at 6 to $10 \mathrm{~kg}$. The results of the

Table I. Least-squares means ( \pm S.E.) of slaughter live weight (kg), empty body weight ( $\mathrm{kg})$, chilling losses (\%) and carcass percentages for Criollo Cordobes and Anglo Nubian kids. (Peso al sacrificio, peso vivo vacío, pérdidas por refrigeración y rendimientos canal en cabritos de las razas Criollo Cordobés y Anglo Nubiana).

\begin{tabular}{|c|c|c|c|c|c|c|c|c|}
\hline \multirow[b]{2}{*}{ Variables } & \multicolumn{3}{|c|}{$\begin{array}{l}\text { Criollo Cordobes } \\
\text { Age at slaughter }\end{array}$} & \multicolumn{3}{|c|}{$\begin{array}{l}\text { Anglonubian } \\
\text { Age at slaughter }\end{array}$} & \multirow{2}{*}{\multicolumn{2}{|c|}{$\begin{array}{l}\text { Effect } \\
\text { B A }\end{array}$}} \\
\hline & Total & Group I & Group II & Total & Group I & Group II & & \\
\hline SLW (kg) & $11.12 \pm 0.19$ & $10.44 \pm 0.36$ & $11.72 \pm 0.24$ & $10.61 \pm 0.15$ & $10.15 \pm 0.18$ & $11.29 \pm 0.15$ & ns & *** \\
\hline EBW (kg) & $9.70 \pm 0.18$ & $9.04 \pm$ & $10.26=$ & $9.27 \pm 0$ & $8.98 \pm$ & $10.04 \pm 0.06$ & $\mathrm{~ns}$ & *** \\
\hline $\mathrm{HCW}$ & $5.34 \pm 0.11$ & $4.98 \pm 0.27$ & $5.65 \pm 0.38$ & $5.43 \pm 0.08$ & $5.18 \pm 0$ & $5.79 \pm 0.06$ & $\mathrm{~ns}$ & *** \\
\hline CCW & $5.22 \pm 0.11$ & $4.86 \pm 0.27$ & $5.51 \pm 0.37$ & $5.29 \pm 0.87$ & $5.05 \pm 0$ & $5.65 \pm 0.18$ & ns & *** \\
\hline $\mathrm{CH}$ & $2.45 \pm 0.14$ & $2.42 \pm 0.22$ & $2.48 \pm 0.1$ & $2.19 \pm 0.16$ & $2.21 \pm 0.17$ & $2.32 \pm 0.13$ & ns & ns \\
\hline HCW/SLW & $48.04 \pm 0.63$ & $47.75 \pm 0.67$ & $48.33 \pm 0.53$ & $51.09 \pm 0.36$ & $50.99 \pm 0.47$ & $51.23 \pm 0.21$ & $* * *$ & ns \\
\hline HCW/EBW & $55.04 \pm 0.54$ & $55.11 \pm 0.32$ & $55.20 \pm 0.63$ & $57.55 \pm 0.14$ & $57.61 \pm 0.30$ & $57.47 \pm 0.17$ & $* \star *$ & ns \\
\hline CCW/ & $46.86 \pm 0.59$ & & $47.13=$ & & & $50.07 \pm 0.24$ & $* \star *$ & ns \\
\hline CCW/EBW & $53.69 \pm 0.51$ & $53.78 \pm 0.34$ & $52.57 \pm 0.69$ & $56.19 \pm 0.22$ & $56.22 \pm 0.29$ & $56.17 \pm 0.16$ & $* * *$ & ns \\
\hline
\end{tabular}

$\mathrm{B}=$ breed; $\mathrm{A}=$ age; $\mathrm{SLW}=$ slaughter liveweight; $\mathrm{EBW}=$ empty body weight; $\mathrm{HCW}=$ hot carcass weight; $\mathrm{CCW}=$ cold carcass weight; $\mathrm{CH}=$ chilling losses $=(\mathrm{HCW}-\mathrm{CCW}) \times 100 / \mathrm{HCW} ; \mathrm{HCW} / \mathrm{SLW}=\mathrm{HCW} * 100 / \mathrm{SLW}$; $\mathrm{HCW} / \mathrm{EBW}=\mathrm{HCW}^{*} 100 / \mathrm{EBW} ; \mathrm{CCW} / \mathrm{SLW}=\mathrm{CCW}^{*} 100 / \mathrm{SLW} ; \mathrm{CCW} / \mathrm{EBW}=\mathrm{CCW}^{*} 100 / \mathrm{EBW}$ ns $=$ no significant; ${ }^{* * *} p<0.001$; group I: 60 days old; group II: 90 days old. 


\section{PEÑA, BONVILLANI, MORANDINI, FREIRE, DOMENECH AND GARCÍA}

present study contrast with the general assertion that while kids consume milk, dressing percentage and their subcutaneous fat increases with the increasing of maturity. In this study, a heavier slaughter weight did not significantly increase $(\mathrm{p}>0.05)$ carcass fatness, this might be the cause of variation between this study and most published papers.

There were significant differences in some carcass measures and indices among breeds and age groups (table II). The results show that the carcasses from AN kids are longer and less compact than the CC kids due to the larger size of the AN breed, in agreement with Dhanda et al. (2003a). Heavier kids have longer carcasses and an improved carcass compactness index, which supports the findings of Peña et al. (2007). The rib eye area, higher than that found by Perez et al. (2001) in Creole kids slaughtered at $8-12 \mathrm{~kg}$ of body weight, showed a significant increase with slaughter weight. Likewise, the average values of ribeye area/ empty body weight ratio were higher $(0.7 \mathrm{vs}$. 0.4-0.5) than those obtained in kids of various genotypes (Dhanda et al., 2003a; Peña et al., 2007).

There were significant differences between breeds for carcass conformation

Table II. Least-squares means ( \pm S.E.) of carcass measures (cm) and indices for Criollo Cordobes and Anglo Nubian kids. (Medidas e índices de la canal en cabritos de las razas Criollo Cordobés y Anglo Nubiana).

\begin{tabular}{|c|c|c|c|c|c|c|c|c|}
\hline \multirow[b]{2}{*}{ Variables } & \multicolumn{3}{|c|}{$\begin{array}{l}\text { Criollo Cordobes } \\
\text { Age at slaughter }\end{array}$} & \multicolumn{3}{|c|}{$\begin{array}{c}\text { Anglonubian } \\
\text { Age at slaughter }\end{array}$} & \multirow{2}{*}{\multicolumn{2}{|c|}{$\begin{array}{c}\text { Effect } \\
\text { B A }\end{array}$}} \\
\hline & Total & Group I & Group II & Total & Group I & Group II & & \\
\hline $\mathrm{F}$ & $28.75 \pm 0.43$ & $28.44 \pm 0.95$ & $28.99 \pm 0.72$ & $29.44 \pm 0.19$ & $29.23 \pm 0.28$ & $30.05 \pm 0.24$ & * & ns \\
\hline $\mathrm{L}$ & $44.62 \pm 0.50$ & $44.28 \pm 0.59$ & $44.86 \pm 0.79$ & $46.22 \pm 0.32$ & $45.48 \pm 0.29$ & $47.33 \pm 0.25$ & $* * *$ & * \\
\hline G & $10.52 \pm 0.13$ & $10.44 \pm 0.18$ & $10.57 \pm 0.12$ & $10.16 \pm 0.14$ & $10.08 \pm 0.16$ & $10.34 \pm 0.09$ & ns & ns \\
\hline $\mathrm{Wr}$ & $11.51 \pm 0.23$ & $11.29 \pm 0.31$ & $11.69 \pm 0.32$ & $11.05 \pm 0.17$ & $10.74 \pm 0.22$ & $11.53 \pm 0.14$ & ns & $* *$ \\
\hline Wth & $10.81 \pm 0.20$ & $10.63 \pm 0.35$ & $10.96 \pm 0.24$ & $10.31 \pm 0.10$ & $10.25 \pm 0.13$ & $10.51 \pm 0.05$ & * & ns \\
\hline Th & $19.75 \pm 0.19$ & $19.23 \pm 0.19$ & $20.28 \pm 0.29$ & $19.17 \pm 0.16$ & $19.09 \pm C$ & $19.33 \pm 0.15$ & ** & ** \\
\hline BG & $37.27 \pm 0.51$ & $36.08 \pm 0.48$ & $38.19 \pm 0.60$ & $37.09 \pm 0.68$ & $35.12 \pm$ & $39.84 \pm 1.18$ & $\mathrm{~ns}$ & *** \\
\hline PT & $48.51 \pm 0.29$ & $47.82 \pm 0$ & $48.88 \pm C$ & $47.45 \pm 0.33$ & 46. & $48.95 \pm 0.35$ & ns & *** \\
\hline A & $3.96 \pm 0.07$ & & & & & .05 & ns & ns \\
\hline B & $2.38 \pm 0$ & 2.2 & 2.4 & $2.34 \pm 0.07$ & $1 \pm 0.08$ & $2.56 \pm 0.06$ & ns & ** \\
\hline REA & $7.31 \pm 0.24$ & 6.5 & 7.58 & 30 & \pm 0.32 & 0.29 & ns & ** \\
\hline L/G & & & & & & & $\star * *$ & ns \\
\hline L/PT & $0.92 \pm 0.01$ & $0.93 \pm 0$ & 0.91 & $0.97 \pm 0.01$ & $0.97 \pm 0.01$ & $0.96 \pm 0.02$ & *** & ns \\
\hline Th/G & $1.87 \pm 0.02$ & $1.85 \pm 0.11$ & $1.92 \pm$ & $1.89 \pm 0.03$ & $1.90 \pm 0.02$ & $1.87 \pm$ & ns & ns \\
\hline $\mathrm{G} / \mathrm{F}$ & $0.37 \pm 0.01$ & $0.37 \pm 0$ & $0.36 \pm 0.01$ & $0.35 \pm 0.01$ & $0.34 \pm 0.02$ & $0.34 \pm 0.01$ & & ns \\
\hline $\mathrm{Wr} / \mathrm{Th}$ & $0.58 \pm 0.03$ & $0.59 \pm 0.02$ & $0.57 \pm 0.04$ & $0.57 \pm 0.01$ & $0.56 \pm 0.02$ & $0.60 \pm 0.03$ & ns & ns \\
\hline Th/L & $0.44 \pm 0.03$ & $0.44 \pm 0.02$ & $0.45 \pm 0.05$ & $0.41 \pm 0.02$ & $0.42 \pm 0.01$ & $0.41 \pm 0.02$ & *** & ns \\
\hline $\mathrm{HCW} / \mathrm{L}$ & $120.25 \pm 2.46$ & $112.49 \pm 1.54$ & $126.31 \pm 3.43$ & $117.89 \pm 1.41$ & $113.89 \pm 1.71$ & $122.62 \pm 1.53$ & $\mathrm{~ns}$ & \\
\hline CCW/L & $117.34 \pm 2.45$ & $109.78 \pm 1.56$ & $123.41 \pm 3.51$ & $114.12 \pm 1.42$ & $111.12 \pm 1.68$ & $119.53 \pm 1.44$ & ns & * \\
\hline
\end{tabular}

$\mathrm{B}=$ breed; $\mathrm{A}=$ age; $\mathrm{F}=$ leg length; $\mathrm{L}=$ internal carcass length; $\mathrm{G}=$ buttock width; Wr= thorax width; Wth= maximum rib width; $T h=$ chest depth; $B G=$ buttock perimeter; $P T=$ thoracic perimeter; $A=$ ribeye width; $B=$ ribeye depth; REA $=$ ribeye area $=(A / 2) \times(B / 2) \times \pi ; H C W / L$ and $C C W / L=$ carcasses compactness $(g /$ $\mathrm{cm}) ; \mathrm{G} / \mathrm{F}=$ leg compactness (G/F); ns= no significant; ${ }^{*} \mathrm{p}<0.05 ;{ }^{* *} \mathrm{p}<0.01$; ${ }^{* * *} \mathrm{p}<0.001$; group I: 60 days old; group II: 90 days old.

Archivos de zootecnia vol. 60, núm. 230, p. 228. 


\section{CARCASS QUALITY OF SUCKLING GOAT KIDS}

and fatness, in agree with Peña et al. (1994) and Oman et al. (2000). AN kids had better $(\mathrm{p}<0.05)$ scores than did $\mathrm{CC}$ kids. However, our data do not agree with Dhanda et al. (2003b) who showed that genotype does not affect carcass conformation. The scores of carcass conformation and subcutaneous fatness were lower than those obtained by Sanz et al. (1995) in kids slaughtered at 2 months old, and the score of muscle colour is lower than that obtained by BecerrilHerrera et al. (2006) in Creole male kids slaughtered at $7 \mathrm{~kg}$ live weight. All the variables increased with slaughter weight, being colour the exception.

Table IV shows the effect of breed and age at slaughter on visceral components. The percentage contribution of fifth quarter were similar to those reported for different breeds at similar live weight (Pérez et al., 2001; Argüello et al., 2007), and different than those obtained by Gallo et al. (1997) in Creole kids slaughtered at higher weight/ age, and by Dhanda et al. (2003b) in different genotypes. CC kids had significantly heavier $(\mathrm{p}<0.05)$ subproducts percentage and lower $(\mathrm{p}<0.05)$ fat depots than AN kids, which might be the reason for the differences in

Table III. Carcass grade scores and percentage of carcasses in each class for Criollo Cordobes and Anglo Nubian kids. (Valoración subjetiva de canales de cabritos de las razas Criollo Cordobés y Anglo Nubiana).

\begin{tabular}{|c|c|c|c|c|c|c|c|c|}
\hline \multirow[b]{2}{*}{ Variables } & \multicolumn{3}{|c|}{$\begin{array}{l}\text { Criollo Cordobes } \\
\text { Age at slaughter }\end{array}$} & \multicolumn{3}{|c|}{$\begin{array}{l}\text { Anglonubian } \\
\text { Age at slaughter }\end{array}$} & \multicolumn{2}{|c|}{ Effect } \\
\hline & Total & Group I & Group II & Total & Group I & Group II & & \\
\hline $\mathrm{CC}$ & $2.08 \pm 0.12$ & $1.88 \pm 0.13$ & $2.18 \pm 0.12$ & $2.42 \pm 0.09$ & $1.71 \pm 0.08$ & $2.70 \pm 0.13$ & * & $* *$ \\
\hline low & 10.0 & 12.45 & 9.09 & 12.32 & 18.54 & - & & \\
\hline medium & 75.00 & 87.55 & 63.64 & 22.54 & 81.46 & 30.00 & & \\
\hline high & 15.00 & - & 27.27 & 65.14 & - & 70.00 & & \\
\hline SF & $1.89 \pm 0.13$ & $1.78 \pm 0.44$ & $2.27 \pm 0.52$ & $3.30 \pm 0.13$ & $3.20 \pm 0.61$ & $3.33 \pm 0.42$ & $* * *$ & ns \\
\hline low & 15.0 & 22.2 & 9.1 & - & - & - & & \\
\hline slight & 65.0 & 77.8 & 54.5 & 4.0 & 6.7 & - & & \\
\hline medium & 20.0 & - & 36.4 & 64.0 & 53.3 & 80.0 & & \\
\hline high & - & - & - & 32.0 & 40.0 & 20.0 & & \\
\hline very high & - & - & - & - & - & - & & \\
\hline IF & $1.37 \pm 0.11$ & $1.44 \pm 0.52$ & $1.36 \pm 0.50$ & $2.70 \pm 0.11$ & $2.73 \pm 0.45$ & $2.70 \pm 0.48$ & $\star * \star$ & ns \\
\hline low & 60.4 & 55.5 & 36.4 & - & - & - & & \\
\hline medium & 40.0 & 45.5 & 63.6 & 28.0 & 30.0 & 26.7 & & \\
\hline high & - & - & - & 72.0 & 70.0 & 73.3 & & \\
\hline SFC & $1.79+0.10$ & $1.55 \pm 0.35$ & $1.89 \pm 0.33$ & $1.65 \pm 0.11$ & $1.60 \pm 0.50$ & $1.80 \pm 0.42$ & ns & ns \\
\hline white & 30.0 & 45.5 & 11.1 & 32.3 & 40.0 & 20.0 & & \\
\hline cream & 70.0 & 55.5 & 88.9 & 67.7 & 60.0 & 80.0 & & \\
\hline yellow & - & - & - & - & - & - & & \\
\hline $\mathrm{MC}$ & $1.58 \pm 0.12$ & $1.44 \pm 0.52$ & $1.82 \pm 0.40$ & $1.60 \pm 0.11$ & $1.67 \pm 0.48$ & $1.30 \pm 0.48$ & ns & ns \\
\hline pale & 35.0 & 55.5 & 18.2 & 48.0 & 70.0 & 33.3 & & \\
\hline pink & 65.0 & 45.5 & 81.8 & 52.0 & 30.0 & 66.7 & & \\
\hline red & - & - & - & - & - & - & & \\
\hline
\end{tabular}

$\mathrm{B}=$ breed; $\mathrm{A}=$ age; $\mathrm{CC}=$ carcass conformation; $\mathrm{SF}=$ subcutaneous fatness; $\mathrm{IF}=$ internal fatness; $\mathrm{SFC}=$ subcutaneous fat colour; $\mathrm{MC}=$ muscle colour; $\mathrm{ns}=$ no significant; ${ }^{* *} \mathrm{p}<0.01 ;{ }^{* *} \mathrm{p}<0.001$; group I: 60 days old; group II: 90 days old. 
carcass percentages (table I).

The fifth quarter, as percentage of EBW, and percentages of skin, subproducts and stomach decreased $(p>0.05)$ and those of the visceral organs and fat depots increased $(p>0.05)$ with increasing EBW. In opposition to that, Marichal et al. (2003) reported a significant increase in the contribution of gastro-intestinal tract as a percentage of the empty body weight; the diet between studies (milk vs. milk + concentrate) could be the main reason for these differences. Peña et al. (2007) recorded a significant decrease in the percentage of internal organs, whereas the percentage of stomach increased. The weight range considered and diet may be the main causes of these differences.

Fat depots percentage was not significantly different $(\mathrm{p}>0.05)$ for age at slaughter. In contrast, Peña et al. (2007) recorded significant increases in the omental and mesenteric fat contents with increased SLW. The level of food and slaughter weight range considered in both studies may be the main causes of the differences.

Cuts of half carcasses arranged from greatest to smallest were: leg $(32 \%)$, ribs (22\%), shoulder (21\%), flank (11\%) and neck (9\%). Grouped by commercial categories, the extra cuts represented $54 \%$, first $21 \%$ and second $20 \%$ of left half carcass weight,

Table IV. Least-squares means ( \pm S.E.) of non-carcass components (\% on empty body weight) of Criollo Cordobes and Anglo Nubian kids. (Quinto cuarto en cabritos de las razas Criollo Cordobés y Anglo Nubiana).

\begin{tabular}{|c|c|c|c|c|c|c|c|c|}
\hline \multirow[b]{2}{*}{ Variables } & \multicolumn{3}{|c|}{$\begin{array}{l}\text { Criollo Cordobes } \\
\text { Age at slaughter }\end{array}$} & \multicolumn{3}{|c|}{$\begin{array}{c}\text { Anglonubian } \\
\text { Age at slaughter }\end{array}$} & \multirow{2}{*}{\multicolumn{2}{|c|}{$\begin{array}{c}\text { Effect } \\
\text { B A }\end{array}$}} \\
\hline & Total & Group I & Group II & Total & Group I & Group II & & \\
\hline NCC & $42.18 \pm 0.45$ & $43.03 \pm 0.47$ & $41.38 \pm 0.65$ & $41.02 \pm 0.19$ & $41.25 \pm 0.23$ & $40.67 \pm 0.29$ & * & ** \\
\hline $\mathrm{Sp}$ & $27.22 \pm 0.93$ & $27.67 \pm 0.27$ & $26.85 \pm 0.79$ & $25.61 \pm 0.23$ & $26.03 \pm 0.27$ & $24.98 \pm 0.32$ & $* * *$ & * \\
\hline Blood & $5.68 \pm 0.65$ & $5.20 \pm 0.22$ & $5.51 \pm 0.28$ & $4.85 \pm 0.08$ & $4.82 \pm 0.14$ & $4.91 \pm 0.12$ & * & ns \\
\hline Skin & $10.90 \pm 0.24$ & $11.49 \pm 0.35$ & $10.41 \pm 0.31$ & $10.72 \pm 0.15$ & $10.98 \pm 0.19$ & $10.33 \pm 0.22$ & ns & * \\
\hline Feet & $3.50 \pm 0.30$ & $3.54 \pm 0.12$ & $3.46 \pm 0.07$ & $3.57 \pm 0.04$ & $3.59 \pm 0.05$ & $3.55 \pm 0.08$ & ns & ns \\
\hline Head & $7.15 \pm 0.19$ & $7.44 \pm 0.15$ & $6.92 \pm 0.12$ & $6.47 \pm 0.08$ & $6.64 \pm 0.09$ & $6.21 \pm 0.09$ & *** & ns \\
\hline VO & $5.10 \pm 0.09$ & $5.19 \pm 0.14$ & $5.03 \pm 0.12$ & $4.98 \pm 0.06$ & $4.92 \pm 0.09$ & $5.08 \pm 0.08$ & ns & ns \\
\hline$L+T$ & $1.77 \pm 0.13$ & $1.74 \pm 0.02$ & $1.79 \pm 0.05$ & $1.65 \pm 0.03$ & $1.65 \pm 0.04$ & $1.64 \pm 0.05$ & * & ns \\
\hline Heart & $0.56 \pm 0.07$ & $0.58 \pm 0.02$ & $0.55 \pm 0.02$ & $0.53 \pm 0.01$ & $0.53 \pm 0.01$ & $0.54 \pm 0.02$ & ns & ns \\
\hline Liver & $2.52 \pm 0.06$ & $2.60 \pm 0.11$ & $2.46 \pm 0.08$ & $2.61 \pm 0.27$ & $2.51 \pm 0.06$ & $2.77 \pm 0.07$ & ns & ns \\
\hline Spleen & $0.24 \pm 0.01$ & $0.26 \pm 0.01$ & $0.23 \pm 0.01$ & $0.24 \pm 0.06$ & $0.23 \pm 0.01$ & $0.25 \pm 0.01$ & ns & ns \\
\hline Stomach & $7.89 \pm 0.21$ & $8.23 \pm 0.26$ & $7.61 \pm 0.32$ & $7.51 \pm 0.09$ & $7.52 \pm 0.13$ & $7.49 \pm 0.14$ & ns & ns \\
\hline Rumen $^{a}$ & $3.12 \pm 0.14$ & $3.12 \pm 0.13$ & $3.13 \pm 0.25$ & $2.99 \pm 0.08$ & $1.99 \pm 0.08$ & $1.99 \pm 0.15$ & $* *$ & ns \\
\hline Intestine & $4.95 \pm 0.23$ & $5.12 \pm 0.28$ & $4.81 \pm 0.36$ & $5.53 \pm 0.08$ & $5.53 \pm 0.13$ & $5.54 \pm 0.07$ & ** & ns \\
\hline Fat depots & $1.98 \pm 0.09$ & $1.91 \pm 0.11$ & $2.05 \pm 0.14$ & $2.82 \pm 0.11$ & $2.78 \pm 0.14$ & $2.88 \pm 0.19$ & *** & ns \\
\hline - Pericardic & $0.08 \pm 0.01$ & $0.09 \pm 0.01$ & $0.08 \pm 0.01$ & $0.10 \pm 0.01$ & $0.10 \pm 0.01$ & $0.10 \pm 0.01$ & ns & ns \\
\hline - Omental & $0.74 \pm 0.06$ & $0.68 \pm 0.08$ & $0.79 \pm 0.11$ & $1.21 \pm 0.07$ & $1.19 \pm 0.09$ & $1.25 \pm 0.12$ & *** & ns \\
\hline - Mesenteric & $1.17 \pm 0.05$ & $1.15 \pm 0.07$ & $1.18 \pm 0.07$ & $1.50 \pm 0.05$ & $1.49 \pm 0.07$ & $1.51 \pm 0.07$ & 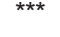 & ns \\
\hline
\end{tabular}

$\mathrm{B}=$ breed; $\mathrm{A}=$ age; $\mathrm{NCC}=$ non-carcass components; $\mathrm{Sp}=$ subproducts; $\mathrm{VO}=$ visceral organs; $\mathrm{L}+\mathrm{T}=$ lungs+trachea; $\mathrm{CCW}=$ cold carcass weight; $\mathrm{HCW}=$ hot carcass weight; $\mathrm{SW}=$ slaughter weight; $\mathrm{EBW}=$ empty body weight;

${ }^{a}$ Rumen= rumen+reticulum+omasum+abomasum; ns= no significant; ${ }^{*} p<0.05 ;{ }^{* *} p<0.01 ;{ }^{* * *} p<0.001$; group I: 60 days old; group II: 90 days old.

Archivos de zootecnia vol. 60, núm. 230, p. 230. 


\section{CARCASS QUALITY OF SUCKLING GOAT KIDS}

respectively. The trend of best yield observed in the cuts is in agreement with the reports of Perez et al. (2001) for Creole goats. The proportion of joint were in the same order as those obtained in previous studies (Monte et al., 2007; Zimmerman et al., 2008) in different goat breed and crosses. However, the percentages of leg and shoulder were higher than those obtained by Perez et al. (2001) in Creole kids reared with goat milk or milk substitute and slaughtered at $9 \mathrm{~kg}$ of EBW. These differences are likely due to different jointing procedures.

There were no significant differences among slaughter weights for the percentage contribution of primal cuts, tail, kidney, thymus, diaphragm, testis and pelvic-renal fat (table V), in agreement with Perez et al. (2001) and Argüello et al. (2007), while the contribution to carcass weight of flank and pelvic-renal fat is higher in AN goat kids, and the percentage of pieces of extra category is lower. Differences between breeds in pelvic-renal fat deposition may be due to the quantity and quality of milk suckled by kids. Pérez et al. (2001) established that milk affects the proportion of pelvic-renal fat.

The correlation between the fat content of the shoulder and the other fatty deposits studied (table VII), yields a high correlation coefficient with the pelvic-renal fat. This fat depot can be a good predictor of carcass fat content, in agreement with Colomer-Rocher etal. (1989).

Means values of percentage of muscle, bone and fat to shoulder weight (table VI) were $65.6 \%, 26.5 \%$ and $6.3 \%$. The lean content was higher to that reported for various other breeds of goat (Oman et al., 2000; Todaro et al., 2004; Zimmerman et al.,

Table $\boldsymbol{V}$. Carcass weight $(\mathrm{kg})$ and percentage contribution, in relation to half-carcass weight, of primal cuts (least-squares means \pm S.E.) of carcasses from Criollo Cordobes and Anglo Nubian kids. (Despiece de la canal de cabritos de las razas Criollo Cordobés y Anglo Nubiana).

\begin{tabular}{|c|c|c|c|c|c|c|c|c|}
\hline \multirow[b]{2}{*}{ Variables } & \multicolumn{3}{|c|}{$\begin{array}{l}\text { Criollo Cordobes } \\
\text { Age at slaughter }\end{array}$} & \multicolumn{3}{|c|}{$\begin{array}{l}\text { Anglonubian } \\
\text { Age at slaughter }\end{array}$} & \multirow{2}{*}{\multicolumn{2}{|c|}{$\begin{array}{l}\text { Effect } \\
\text { B A }\end{array}$}} \\
\hline & Total & Group I & Group II & Total & Group I & Group II & & \\
\hline Left side weight & $2.56 \pm 0.0 .05$ & $2.41 \pm 0.06$ & $2.69 \pm 0.05$ & $2.56 \pm 0.04$ & & & ns & \\
\hline Leg \% & $32.45 \pm 0.32$ & $31.84 \pm 0$ & & & $31.82 \pm 1.06$ & & ns & \\
\hline Shoulder \% & $21.69 \pm 0.27$ & $21.39 \pm 0.33$ & & $20.89 \pm 0.24$ & & & ns & ns \\
\hline Ribs \% & $22.76 \pm 0.29$ & 22.9 & & & & $22.17 \pm 1.74$ & $\mathrm{~ns}$ & ns \\
\hline & & & & & & & ns & ns \\
\hline & 10. & & & & & & & ns \\
\hline & & & & & & & $* * *$ & \\
\hline & & & & & & & ns & ns \\
\hline & & & & & & & $\star \star * *$ & \\
\hline Dia & & & & & & & $\mathrm{ns}$ & \\
\hline nal fat $\%$ & $1.15 \pm 0.11$ & & & & & 4.3 & $\star \star * *$ & \\
\hline $\begin{array}{l}\text { Testis \% } \\
\text { By categories }\end{array}$ & $0.83 \pm 0.04$ & $0.76 \pm 0.05$ & $0.88 \pm 0.04$ & $0.64 \pm 0.02$ & $0.65 \pm 0.02$ & $0.62 \pm 0.04$ & $* * *$ & \\
\hline Extra & $55.23 \pm 0.27$ & & & & & & ${ }^{* *}$ & $\mathrm{~ns}$ \\
\hline & $21.69 \pm 0.28$ & $21.39 \pm 0.33$ & $21.93 \pm 0.42$ & $20.89 \pm 0.21$ & $20.89 \pm 0.27$ & $20.90 \pm 0.36$ & ns & ns \\
\hline Second & $19.85 \pm 0.31$ & $20.02 \pm 0.47$ & $19.71 \pm 0.45$ & $20.24 \pm 0.26$ & $20.52 \pm 0.33$ & $19.82 \pm 0.39$ & $\mathrm{~ns}$ & ns \\
\hline
\end{tabular}

$B=$ breed; $A=$ age; $n s=$ no significant; ${ }^{*} p<0.05 ;{ }^{* * *} p<0.001$; group I: 60 days old; group II: 90 days old. 
Table VI. Tissue composition of the shoulder from Criollo Cordobes and Anglo Nubian kids (Least-squares means $\pm S$.E.). (Composición tisular de la espalda en cabritos de las razas Criollo Cordobés y Anglo Nubiana).

\begin{tabular}{|c|c|c|c|c|c|c|c|c|}
\hline \multirow[b]{2}{*}{ Variables } & \multicolumn{3}{|c|}{$\begin{array}{l}\text { Criollo Cordobes } \\
\text { Age at slaughter }\end{array}$} & \multicolumn{3}{|c|}{$\begin{array}{c}\text { Anglonubian } \\
\text { Age at slaughter }\end{array}$} & \multirow{2}{*}{\multicolumn{2}{|c|}{$\begin{array}{l}\text { Effect } \\
\text { B A }\end{array}$}} \\
\hline & Total & Group I & Group II & Total & Group I & Group II & & \\
\hline Muscle & $66.29 \pm 0.72$ & $66.91 \pm 0.38$ & $65.80 \pm 1.30$ & $64.87 \pm 0.35$ & $65.07 \pm 0.51$ & $64.97 \pm 0.46$ & * & ns \\
\hline Bone & $26.00 \pm 0.61$ & $26.89 \pm 0.82$ & $25.27 \pm 0.84$ & $27.09 \pm 0.36$ & $27.52 \pm 0.56$ & $27.18+0.35$ & $\mathrm{~ns}$ & ns \\
\hline Dissectible fat & $5.68 \pm 0.34$ & $5.68 \pm 0.51$ & $5.68 \pm 0.47$ & $6.87 \pm 0.56$ & $6.77 \pm 0.49$ & $6.72 \pm 0.41$ & ns & ns \\
\hline Other tissues & $1.09 \pm 0.10$ & $1.03 \pm 0.15$ & $1.15 \pm 0.14$ & $1.10 \pm 0.07$ & $1.02 \pm 0.10$ & $1.05 \pm 0.08$ & ns & ns \\
\hline Muscle/fat & $12.57 \pm 0.69$ & $12.55+0.99$ & $12.49 \pm 0.99$ & $9.68 \pm 0.63$ & $9.80 \pm 0.98$ & $9.48 \pm 0.63$ & $\star *$ & ns \\
\hline Muscle/bone & $2.58 \pm 0.07$ & $2.51 \pm 0.08$ & $2.63 \pm 0.12$ & $2.39 \pm 0.04$ & $2.38 \pm 0.05$ & $2.42 \pm 0.04$ & * & ns \\
\hline
\end{tabular}

$\mathrm{B}=$ breed; $\mathrm{A}=$ age; $\mathrm{ns}=$ no significant; ${ }^{*} \mathrm{p}<0.05 ;{ }^{* *} \mathrm{p}<0.01 ;{ }^{* * *} \mathrm{p}<0.001$; group I: 60 days old; group II: 90 days old.

2008) whilst the dissectible fat was lower. Bone carcass proportions also differed from the values found by Marichal et al. (2003) and Peña et al. (2007) in kids slaughtered at similar carcass weight, respectively. The lower fat content could be explained by the low nutritional level of the diets of goats and therefore their kids (Oman et al., 2000).
Monte et al. (2007) working with AN crosses kids slaughtered at $14 \mathrm{~kg}$ carcass weight recorded mean values of contribution to shoulder weight of $62 \%, 15 \%, 8 \%$ for muscle, bone and fat, respectively. Dhanda et al. (1999) recorded percentages of $62-64 \%$ for muscle, $5-7 \%$ for fat and $24-28 \%$ for bone in five goat crosses slaughtered as Capretto.

Table VII. Pearson correlation values between empty body weight, carcass compactness and tissue distribution. (Correlaciones entre peso vivo vacío, compacidad de la canal y composición tisular de la canal).

\begin{tabular}{|c|c|c|c|c|c|c|c|c|c|c|c|}
\hline & EBW & $\mathrm{HCW} / \mathrm{L}$ & M & B & $\mathrm{F}$ & P.F. & O. F. & M. F. & $F-Q F$. & P-R.F. & $M / F$ \\
\hline HCW/L & $0.78^{* *}$ & & & & & & & & & & \\
\hline Muscle & ns & ns & & & & & & & & & \\
\hline Bone & ns & ns & ns & & & & & & & & \\
\hline Fat & ns & ns & ns & ns & & & & & & & \\
\hline Pericardic fat & ns & ns & ns & ns & $0.41^{*}$ & & & & & & \\
\hline Omental fat & ns & ns & ns & ns & $0.69^{* *}$ & $0.65^{\star *}$ & & & & & \\
\hline Mesenteric fat & ns & ns & ns & ns & $0.32^{*}$ & $0.41^{*}$ & $0.55^{\star}$ & & & & \\
\hline fifth-quart fat & ns & ns & $\mathrm{ns}$ & $\mathrm{ns}$ & $0.62^{* *}$ & $0.65^{\star *}$ & $0.92^{* * *}$ & $0.83^{* * *}$ & & & \\
\hline Pelvic-renal fat & ns & ns & ns & ns & $0.54^{*}$ & $0.53^{*}$ & $0.80^{* * *}$ & $0.56^{*}$ & $0.82^{* * *}$ & & \\
\hline $\mathrm{M} / \mathrm{F}$ & ns & ns & ns & $\mathrm{ns}$ & $-0.85^{\star * *}$ & $-0.41^{*}$ & $-0.59^{* *}$ & $-0.30^{*}$ & $-0.53^{*}$ & $-0.48^{*}$ & \\
\hline M/B & ns & ns & $0.52^{*}$ & $-0.92^{\star * *}$ & ns & ns & ns & $\mathrm{ns}$ & ns & ns & ns \\
\hline
\end{tabular}

$\mathrm{EBW}=$ empty body weight; $\mathrm{HCW} / \mathrm{L}=$ carcass compatness; $\mathrm{M}=$ muscle; $\mathrm{B}=$ bone; $\mathrm{F}=$ fat; $\mathrm{P} . \mathrm{F}=$ pericardic fat; O.F.= omental fat; M.F.= mesenteric fat; F-Q F.= fifth-quart fat; P-R.F.= pelvic-renal fat; $M / F=$ muscle/ fat; $M / B=$ muscle/bone; ${ }^{*} p<0.05 ;{ }^{* *} p<0.01 ;{ }^{* * *} p<0.001$; ns $=$ not significant.

Archivos de zootecnia vol. 60, núm. 230, p. 232. 


\section{CARCASS QUALITY OF SUCKLING GOAT KIDS}

We found more similarities with the results recorded by Dhanda et al. (2003b), Argüello et al. (2007), and Santos et al. (2007).

One of the reasons for the differences in tissue composition may be the joint of reference (Gallo et al., 1997; Zimmerman et al., 2008). Another reason may be the slaughter weight and diet: high vs. low weights and high energy vs. low energy diets alter the composition tissue. Genotype may be another cause of the differences in the tissue composition of carcass. Comparing goats of different mature body sizes at the same live weight may indicate differences which are related to the stages of maturity rather than breed per se (Dhanda et al., 2003b).

Distribution of carcass tissues is important in determining carcass quality. Breed influenced $(p<0.05)$ the muscle content, muscle/fat and muscle/bone ratios. $\mathrm{CC}$ kids had higher values than AN kids at the same carcass weight. These differences can be partially attributed to mature body size (Mahgoub and Lu, 1998), which is higher in AN goat breed. These results show that carcasses from CC kids, at similar slaughter weight, are leaner compared with carcasses of AN kids.

There were no significant $(\mathrm{p}>0.05)$ differences among slaughter weight in percentage of muscle, bone, fat and remainder, despite of a higher assessment of subjective subcutaneous fatness (table III). However, the contribution of muscle and bone on shoulder weight tends to be lower when slaughter weight, while fat remains more or less constant over the various slaughter weights. These results do not support the findings of Dhanda et al. (1999) and Peña et al. (2007). The muscle/ bone ratio tended to be higher and the muscle/fat ratio lower when SLW weight of kids was high. The early deposition of bone and muscle in relation to fat, might be the reason for these results.

The rate of maturity of CC kids is higher than for AN kids, which is why they produce a greater dissected fat of shoulder/pelvicrenal fat ratio in comparison with the AN kids (19.5 vs. 7.6). These values also confirm that goats tend to store a great portion of their fat internally, rather than in subcutaneous+intermuscular sites (Zimmerman et al., 2008).

The internal fat/carcass fat ratio was $9.37 \pm 0.69$ in CC kids, and increased with slaughter weight $(8.57 \pm 0.79$ in group I and $10.01 \pm 1.06$ in group II), in agreement Gibb et al. (1993) who suggest that dairy goat breeds tend to store more fat in visceral depots, rather than as carcass adipose tissue. In AN kids was $12.15 \pm 0.76,11.45 \pm$ 1.14 and $13.20 \pm 0.77$, respectively. The subcutaneous fat developing later than pelvic-renal fat produces a decrease of subcutaneous fat/pelvic-renal fat ratio with increasing slaughter weight $(20.92 \pm 2.00 \mathrm{vs}$. $18.41 \pm 1.66$ in CC kids and $8.33 \pm 0.84$ vs. 6.56 \pm 0.87 in AN kids).

Table VII shows the principal correlation between empty body weight, carcass compactness and tissue distribution. The carcass conformation was not significantly correlated with any on the traits studied, except for empty body weight. These results are in disagreement with those pointed by Marichal et al. (2003), who noted that carcass with high conformation present more fat, lower bone content and better muscle/ bone ratio.

\section{CONCLUSIONS}

$\mathrm{CC}$ and AN goats are breeds with characteristics which make them suitable for profitable meat production. Within the weight range considered, their lean is pink and their carcasses have a good conformation and an adequate fatness. The higher proportion of muscle and moderate proportion of carcass fat (subcutaneous + intermuscular) indicate the potential of the both breeds as a source of lean meat.

Results obtained in this study showed that breed and slaughter weight influenced 


\section{PEÑA, BONVILLANI, MORANDINI, FREIRE, DOMENECH AND GARCÍA}

some carcass traits. The Anglo Nubian kids produced carcasses with better conformation and higher fatness; whereas the carcasses from Criollo Cordobes kids have a higher content in muscle and lower proportion of fat.

The carcass compactness index and subjective carcass conformation increased significantly and fifth quarter decreased as slaughter weight increased. However, the proportion of cut and tissue composition of the shoulder do not differ.

It would seem from the data presented that there is some advantage to be gained in terms of dressing out percentage, carcass compactness and yield of edible visceral, if male $\mathrm{CC}$ kids and $\mathrm{AN}$ kids are slaughterer at a higher live weight. However there is little

\section{REFERENCES}

Arias, M. and Alonso, A. 2002. Estudio sobre sistemas caprinos del norte de la provincia de Córdoba. Argentina. Arch. Zootec., 51: 341349 .

Argüello, A., Castro, N., Capote, J. and Solomon, M.B. 2007. The influence of artificial rearing and live weight at slaughter on kid carcass characteristics. J. Anim. Vet. Adv., 6: 20-25.

Becerril-Herrera, M., Guzmán-Pina, O., AlonsoSpilsbury, M., Dorsey-San Vicente, E.V., LemusFlores, C., Flores-Peinado, S., RamírezNecoechea, R. and Mota-Rojas, D. 2006. Morphometry, carcass yield and traits of Mexican Creole goat kids slaughtered and packet in a federal inspection. Plant. J. Biolog. Sci., 6: 604609.

Boccard, D.R., Dumont, B.L. et Peyron, C. 1958. Valeur significative de quelques mensurations pour aprecier la qualité des carcasses d'agneux (significant value of same meausurements to appreciate the quality of the lambs carcass). In: Proceedings of the $4^{\text {th }}$ meeting of the European research workers. Cambridge. pp. 15-19.

Colomer-Rocher, F., Morand-Fehr, P. and Kirton, A.H. 1987. Standard methods and procedures for goat carcass evaluation, jointing and tissue separation. Livest. Prod. Sci., 17: 149-159.

Colomer-Rocher, F., Delfa, R. y Echiguer, M. 1989. or no advantage in terms of carcass jointing and carcass composition. The cost of growing the goats to higher weights is greater, and generally their price is lower. From these results it is recommended male kids to be slaughtered at 60 days old. The highest fatness of AN kids allows slaughter at lower live weights than those CC kids.

\section{ACKNOWLEDGEMENTS}

We are grateful to Mr. Omar Montoya for their technical assistance. Financial support for this study by the Secretary of Science and Technology of National University of Rio Cuarto and the National Agency for Scientific and Technological Promotion (Argentina) is gratefully acknowledged.

Características cuantitativas y cualitativas de las canales de los cabritos de raza MurcianoGranadina. Em: $2^{\circ}$ Encontro dos Engenheiros Zootecnicos. Vila Real (Portugal). pp. 125-137.

De Gea, G., Petryna, A.M., Mellano, A., Bonvillani, A. y Turiello, P. 2005b. Características de la canal y de la carne en cabritos tipo Criollo. En: El ganado caprino en la Argentina. Universidad Nacional de Rio Cuarto. Argentina. pp. 3-37.

Dhanda, J.S., Taylor, D.G., McCosker, J.E. and Murray, P.J. 1999. The influence of goat genotype on the production of Capretto and Chevon carcasses. 3. Dissected carcass composition. Meat Sci., 52: 369-374.

Dhanda, J.S., Taylor, D.G. and Murray, P.J. 2003a. Part I. Growth, carcass and meat quality parameters of male goats: effects of genotype and live weight at slaughter. Small Ruminant Res., 50: 57-66.

Dhanda, J.S., Taylor, D.G. and Murray, P.J. 2003b. Part II. Carcass composition and fatty acid profiles of adipose tissue of male goats: effects of genotype and live weight at slaughter. Small Ruminant Res., 50: 67-74.

Domingo, E., Abad, M., Lanari, M.R. y Bidinost, F. 2008. Características de las canales del caprino Criollo del Neuquén. Arch. Zootec., 58: 361-364. Gallo, C., Le Breton, Y., Wainnright, I. and Berkhoff, 


\section{CARCASS QUALITY OF SUCKLING GOAT KIDS}

M. 1997. Body and carcass composition of male and female Criollo goats in the South of Chile. Small Ruminant Res., 23: 163-169.

Gibb, M.J., Cook, J.E. and Treacher, T.T. 1993. Performance of British Saanen, Boer x British Saanen and Anglo-Nubian castrated male kids from 8 weeks to slaughter at 28, 33 or $38 \mathrm{~kg}$ live weight. Anim. Prod., 57: 263-271.

Mahgoub, O. and Lu, C.D. 1998. Growth, body composition and carcass tissue distribution in goats of large and small sizes. Small Ruminant Res., 27: 267-278.

Marichal, A., Castro, N., Capote, J., Zamorano, M.J. and Argüello, A. 2003. Effects of live weight at slaughter $(6,10$ and $25 \mathrm{~kg})$ on kid carcass and meat quality. Livest. Prod. Sci., 83 247-256.

Maubecin, R. 1973. La explotación del ganado caprino en la República Argentina. Información Técnica $n^{\circ} 55$, INTA EEA. Manferedi. Córdoba. Argentina. 23 pp.

Maubecin, R.A. 1976. Modelo de producción caprina para el noroeste de Córdoba. Información Técnica ${ }^{\circ}{ }^{6} 68$. INTA. Estación Experimental Manferedi. Córdoba. Argentina. 14 pp.

Meneses, R.R., Rojas, A.O., Flores, H.P. and Romero, O.Y. 2004. Rendimientos y composición de canales de cabritos Criollos e híbridos Cashmere. Arch. Zootec., 53: 107-110.

Monte, A., Selaive-Villaroel, A., Pérez, J., Zapata, F., Beserra, J. and Nunes de Oliveira, A. 2007. Rendimento de cortes comerciais e composiçao da carcaça de cabritos mestiços. Rev. Bras. Zootecn., 36: 2127-2133.

Oman, J.S., Waldron, D.F., Griffin, D.B. and Savell, J.W. 2000. Carcass traits and retail display-life of chops from different goat breed types. J. Anim. Sci., 78: 1262-1266.

Owen, J.E., Arias Cereceres, M.T. and García Macías, J.A. 1982. The effects of body weight on carcass development of Criollo goats in Northern Mexico. In: $3^{\text {rd }}$ Int. Conference on Goat Production and Disease. Tucson. Arizona. 10: 2427.

Palsson, H. 1939. Meat qualities in the sheep with special reference to Scottish breed and crosses. Carcass measurements and sample joints as indices of quality and composition. J. Agric. Sci., 24: 544-574.

Peña, F., Gutiérrez, M.J., Herrera, M. y Rodero, E. 1994. Clasificación de canales de cabritos de raza Florida Sevillana. Arch. Zootec., 43: 45-53.

Peña, F., J. Perea, A. García and R. Acero. 2007. Effects of weight and sex on the carcass characteristics of Florida suckling kids. Meat Sci., 75: 543-550.

Pérez, P., Maino, M., Morales, M.S. and Soto, A. 2001. Effect of goat milk substitutes and sex on productive parameters and carcass composition of Creole kids. Small Ruminant Res., 42: 87-93.

Rossanigo, C.E., Frigerio, K.L. y Silva Colomer, J. 1995. La cabra Criolla Sanluiseña. Información Técnica $n^{\circ} 135$. EEA. INTA. Centro Regional La Pampa-San Luís. San Luís. Argentina. 21 pp.

Santos, V.A.C., Silva, A.O., Cardoso, J.V.F., Silvestre, A.J.D., Silva, S.R., Martins, C. and Azevedo, J.M.T. 2007. Genotype and sex effects on carcass and meat quality of suckling kids protected by the PGI "Cabrito de Barroso". Meat Sci., 75: 725-736.

Sanz, M.R., Ruiz, I., Gil, F. and Boza, J. 1995. Classification and composition of the carcass of pre-ruminant kid goats of the Granadina breed. Serie A: seminaires mediterraneens. CIHEAM. 197-202.

SAS. 1997. SAS user'guide (release 8.1). Statistical Analysis System, SAS Institute Inc. Cary, NC.

Todaro, M., Corrao, A., Alicata, M.L., Schinelli, R., Giaccone, P. and Priolo, A. 2004. Effects of litter size and sex on meat quality traits of kid meat. Small Ruminant Res., 54: 191-196.

Yañez, E.A., Ferreira, A.C.D., Medeiros, A.N., Pereira Filho, J.M., Teixeira, A. and Resende, K.T. 2006. Methodologies for ribeye area determination in goats. Small Ruminat Res., 63: 197-200.

Zimmerman, M., Domingo, E. and Lanari, M.R. 2008. Carcass characteristics of Neuquén Criollo kids in Patagonia region, Argentina. Meat Sci., 79: 453-457. 